\title{
CROSSOVER DYNAMICS IN BOND DISORDERED LATTICES
}

\author{
G.A. VAN VELZEN and Matthieu H. ERNST \\ Institute of Theoretical Physics, University of Utrecht, 3508 TA Utrecht, The Netherlands \\ James W. DUFTY \\ Department of Physics, University of Florida, Gainesville, FL 32611, USA
}

Received 3 August 1988

\begin{abstract}
The asymptotic dynamics of the percolation model for a bond disordered lattice is studied. The velocity autocorrelation function (VACF) is investigated for arbitrary concentration of disorder in two and three dimensions using an effective medium approximation (EMA). Corrections to the long time tails away from the percolation threshold and to the percolation tails at the threshold are calculated. A characteristic time scale for the long time tails is identified and found to diverge at the threshold. Sufficiently close to the threshold the two types of asymptotic dynamics can be identified clearly for times greater than and less than this characteristic time, respectively. An approximate scaling of the EMA equation is obtained near the threshold for investigation of the crossover region. More generally, the EMA equation is solved numerically for arbitrary concentration in two dimensions to exhibit the complete time dependence of the VACF in all domains near and far from the threshold.
\end{abstract}

\section{Introduction}

Random walks on disordered lattices provide models for the dynamics of a wide variety of different physical systems. Because of this broad interest a great deal of attention has been given to their analysis, as indicated in two recent reviews $[1,2]$. The objective here is to explore the long time dynamics of disordered lattices, which exhibit many of the qualitative features of long time dynamics in real fluids [3] and in diffusive systems with static disorder [4] such as the Lorentz gas. Of particular interest is the slow power law decay of correlation functions, such as the velocity autocorrelation function (VACF), with asymptotic behavior like $t^{-d / 2}$ for fluids and $t^{-1-d / 2}$ for systems with static disorder in dimensions $d \geqq 2$. These "long time tails" are caused by slow collective modes of the system, such as hydrodynamic or diffusive modes.

A strong motivation for this investigation was the computer simulation results of Alder and Alley [5] on the two dimensional Lorentz gas of overlap- 
ping circular scatterers with radius $R$. This is a continuum percolation model with a threshold at a dimensionless density $c_{0} \sim 0.37$, where $c=n R^{2}$. These authors found a long time tail in the VACF $\sim t^{-\alpha(c)}$ with an exponent decreasing above the threshold from $\alpha(c) \sim 2$ at $c \sim 0.03$ to a minimum 1.34 at the percolation threshold and increasing again below threshold to 2.1 at $c \sim 0.74$. We propose to interpret this apparent concentration dependence of the exponent more fundamentally as a crossover between two types of asymptotic dynamics, each characterized by a different exponent [6]. One of these is the universal long time tail indicated above with an exponent denoted by $b_{0}=$ $1+d / 2$, prominent at low densities away from the threshold. The other represents percolation dynamics that applies at the threshold with a different exponent, denoted by $b$. Our primary support for this idea comes from the behavior of VACF and the mean square displacement $\left\langle r^{2}\right\rangle$ for lattice percolation models. The existence of the long time tails $\sim t^{-1-d / 2}$ for the VACF in these models above the threshold has been established by mode coupling theories [4], exact low density calculations [7-9], effective medium theories $[10-15]$ and computer simulations $[16,17]$. More extensive studies have been made of diffusion in the close vicinity of the percolation threshold, and we briefly quote the necessary results from a recent review [2]. Let $c$ denote the fraction of non-conducting bonds or sites (called "hard scatterers" when interpreted as a Lorentz gas) and let $\varepsilon=\left(c_{0}-c\right) / c_{0}$ be the relative distance from the percolation threshold, $c_{0}$. Then the large time behaviour of the mean square displacement is given by

$$
\left\langle r^{2}\right\rangle(t, \varepsilon) \sim t|\varepsilon|^{x} F\left(|\varepsilon|^{y} t\right) \sim \begin{cases}t \varepsilon^{x}, & \varepsilon>0, \\ t^{1-x / y}, & \varepsilon=0 \\ |\varepsilon|^{x-y}, & \varepsilon<0\end{cases}
$$

where $x=\mu$ is the conductivity exponent and $x-y=\beta-2 \nu$ (see ref. [18]). The exponent of $\left\langle r^{2}\right\rangle$ below the threshold is frequently expressed in terms of the fractal dimension, $d_{\mathrm{w}}^{\prime}$ of the random walk, namely $1-x / y=2 / d_{\mathrm{w}}^{\prime}$ (see chapter 2 of ref. [2]). The values of these exponents are summarized in table I. These results imply that the VACF at threshold behaves as $t^{-b}$ with $b=$ $1+x / y$, where $b \simeq 1.34$ for two-dimensional lattices. This is about the same value as found by Alder and Alley near threshold for the overlapping planar Lorentz gas. For the Swiss cheese or the continuum void percolation models in two dimensions, table I gives the approximate value $b \simeq 1.2$. These results suggest that the features of the lattice models studied here may have a qualitative similarity to the continuum percolation model of a Lorentz gas with overlapping scatterers.

Here, we study the detailed behavior and crossover of the VACF above, at, and below threshold. The model considered is a bond disordered lattice with 
Table I

Percolation exponents.

\begin{tabular}{lllllll}
\hline & $\beta$ & $\nu$ & $x$ & $y$ & $d_{\mathrm{w}}^{\prime}$ & $b$ \\
\hline $\begin{array}{l}\text { Lorentz gas }[5] \\
d=2\end{array}$ & & & & & & \\
Lattice [2] & & & & & & 1.34 \\
$d=2$ & $\frac{5}{36}$ & $\frac{4}{3}$ & 1.3 & 3.8 & 3.0 & 1.34 \\
$d=3$ & 0.44 & 0.88 & 2.1 & 3.4 & 4.9 & 1.59 \\
Swiss cheese [2] & & & & & & \\
$d=2$ & $\frac{5}{36}$ & $\frac{4}{3}$ & $0.5-0.7$ & 3.1 & $2.3-2.6$ & $1.17-1.24$ \\
$d=3$ & 0.44 & 0.88 & $2.4-2.6$ & 3.8 & $5.6-5.7$ & $1.64-1.65$ \\
Mean field [12] & & & & & & \\
& 1.0 & $\frac{1}{2}$ & 3 & 3 & & 2 \\
EMA [18] & & & & & & \\
& & & 1 & 2 & 4 & $\frac{3}{2}$ \\
\hline
\end{tabular}

percolation, which is a type of lattice Lorentz gas. Recently, this problem has been studied in detail at low concentration [7-9]. The VACF has been determined for all times exactly through second order in the concentration. The expected long time tails are obtained with coefficients determined exactly to this order in the concentration. However, for concentrations near percolation, the exponent for asymptotic decay is different from that for the long time tails, indicating a crossover between two types of dynamics as the concentration approaches its critical value.

To investigate this problem at these higher concentrations, a more phenomenological approach is required. A relatively simple and accurate description is given by an effective medium approximation (EMA), which has been applied to bond percolation problems on lattices by many authors [10-15]. However, the interplay and crossover between long time dynamics and percolation dynamics in the VACF, which is particularly strong in two dimensions, has hardly been considered in the literature [22]. A closely related problem is the crossover near the percolation threshold in the spectral density of disordered lattices from phonon-type behavior at small frequencies (analogous to long time dynamics) to fracton type behavior at large frequencies (analogous to percolation dynamics). Using the EMA, Derrida et al. [13] have calculated the complete scaling function $F(x)$ in eq. (1.1). However, they did not address the topic of this paper, namely the crossover in the VACF from percolation dynamics to long time tails. The main objective here is to give a rather complete description of this phenomenon, both analytically and numerically, as a function of the concentration. 
In the next section, the model is defined and the long time behavior for the VACF is given above, at, and below the threshold. In the percolating domain, the long time tails plus their subleading corrections are calculated, while in the non-percolating domain a more rapidly decaying dynamics is characterized. In both cases the amplitudes diverge as the threshold is approached, indicating that the expansions are not uniform with respect to the concentration. The subleading terms allow identification of a characteristic time scale as a function of the concentration to define the domain of validity of these expansions. The time scale diverges at the threshold, implying that the domain for the long time tails is shifted to infinite times. At large but finite times, the VACF exhibits a different algebraic tail characteristic of the dynamics at the percolation threshold. Three distinct domains of dynamics are identified corresponding to short time behavior, intermediate time percolation dynamics, and long time tails. The size and location of each domain depends on the concentration; sufficiently close to the threshold each can be identified clearly, while far from the threshold the percolation dynamics domain vanishes.

In section 3, the EMA is written in a scaled form appropriate for concentrations near the threshold. There is no exact scaling in either two or three dimensions to represent all three domains. However, for dimensionality $d>2$ the EMA results for the VACF in the vicinity of the threshold can be represented in a scaling form $|\varepsilon|^{x} F_{0}\left(|\varepsilon|^{y} t\right)$ for the percolation dynamics and in a correction to scaling $|\varepsilon|^{x^{\prime \prime}} F_{1}\left(|\varepsilon|^{y} t\right)$, with $x^{\prime}<x^{\prime \prime}$, for the long time tails. Thus, near the threshold the long time domain vanishes relative to the percolation dynamics for $d>2$. In two dimensions, where the exponents $x^{\prime}$ and $x^{\prime \prime}$ are equal, this is no longer the case. Although the scaling is not exact, the residual dependence on concentration is only logarithmic.

In section 4, the crossover behavior of the VACF and its Fourier transform is presented over a wide range of times and frequencies for the two-dimensional case. The results are obtained by numerical solution of the complete EMA equation both above and below threshold for a range of concentrations near and far from the critical value. The transition between the various asymptotic domains is clearly demonstrated as a function of time, frequency and concentration. Finally, in section 5, the EMA results for the VACF are summarized in terms of scaling laws and corrections to scaling. Also, some connection with other theoretical results and computer simulations is noted.

\section{Effective medium approximations}

A general class of disordered lattice problems can be described by a Markovian process with a master equation of the form 


$$
\frac{\mathrm{d}}{\mathrm{d} t} p_{n}(t)=\sum_{m} L_{n m} p_{m}(t)=\sum_{m}\left[w_{n m} p_{m}(t)-w_{m n} p_{n}(t)\right],
$$

where $p_{n}(t)$ is the probability for occupation of site $n$ at time $t$. The transition rates $w_{n m}$ from lattice site $m$ to site $n$ are nonzero only for nearest neighbors. They depend on one or more random variables that represent the statistical disorder of the lattice. The lattice is taken to be a $d$-dimensional cubic lattice with unit lattice distance and periodic boundary conditions. The $N$ sites are labeled by $n=\left(n_{1}, n_{2}, \ldots, n_{d}\right)$ and their nearest neighbors are labeled by $n+\rho$. The percolation model for a bond disordered lattice is obtained by removing at random a fraction, $c$, of the bonds $(n, n+\rho)$. Then the transition rates are random variables with a bond-independent probability distribution,

$$
w_{n, n+\rho}= \begin{cases}0, & \text { with probability } c, \\ 1 / 2 d, & \text { with probability } 1-c .\end{cases}
$$

One of the most important dynamical properties of the disordered lattice is the $\mathrm{VACF}$, defined in terms of the mean square displacement by

$$
\begin{aligned}
V(t) & =\frac{1}{2}\left(\mathrm{~d}^{2} / \mathrm{d} t^{2}\right)\left\langle r^{2}\right\rangle(t) \\
& =\frac{1}{2}\left(\mathrm{~d}^{2} / \mathrm{d} t^{2}\right) N^{-1} \sum_{n, m}\left(n_{x}-m_{x}\right)^{2}\langle p(n, t \mid m)\rangle .
\end{aligned}
$$

Here $p(n, t \mid m)$ is the solution to (2.1) with $p(n, 0 \mid m)=\delta_{n m}$. The brackets \langle\rangle denote an average over the random variables, $w_{n, n+\rho}$. It is convenient to introduce a function $\Psi(z)$ that is proportional to the Laplace transform of the VACF,

$$
\Psi(z)=2 d \int_{0}^{\infty} \mathrm{d} t \mathrm{e}^{-z t} V(t) .
$$

In the EMA the function $\Psi(z)$ is determined from the self-consistency requirement (see eq. (6.80) of ref. [1])

$$
\left\langle\Delta_{n, n+\rho} /\left[1-R(z) \Delta_{n, n+\rho}\right]\right\rangle=0,
$$

where

$$
\begin{aligned}
& \Delta_{n, n+\rho}=\Psi(z)-2 d w_{n, n+\rho}, \\
& R(z)=(d \Psi)^{-1}\{1-(z / \Psi) L(z / \Psi)\} .
\end{aligned}
$$


Here $L(z)$ is the single site lattice Green's function for a $d$-dimensional simple cubic lattice,

$$
\begin{aligned}
& L(z) \equiv(2 \pi)^{-d} \int_{-\pi}^{\pi} \cdots \int_{-\pi}^{\pi} \mathrm{d}^{(d)} q[z+\omega(q)]^{-1}, \\
& \omega(q) \equiv \frac{1}{d} \sum_{\rho}[1-\cos (\rho q)],
\end{aligned}
$$

where $\rho q=\boldsymbol{\rho} \cdot \boldsymbol{q}$ is an inner product. After some rearrangements, eq. (2.5) reduces to

$$
d \Psi(1-c-\Psi)=(1-\Psi)\{\Psi-z L(z / \Psi)\}
$$

Although approximate, the EMA preserves several exact properties. The diffusion coefficient, defined by $D=\Psi(0) / 2 d$, is given in the EMA by

$$
D=\frac{1}{2 d} \times \begin{cases}\varepsilon, & c<c_{0} \\ 0, & c>c_{0}\end{cases}
$$

where $\varepsilon \equiv\left(c_{0}-c\right) / c_{0}$ and $c_{0} \equiv(d-1) / d$ is the percolation concentration. This value for $c_{0}$ is exact for the square lattice in $d=2$. The expansion of $\Psi(z)$ in powers of the concentration is exact for all $z$ up through first order in $c$, and gives a very good approximation for the second order coefficient. Also, an expansion in $z^{-1}$ is exact for all $c$ up through second order [9]. These results indicate that the EMA might be a reasonable approximation over the whole parameter space, particularly for $d=2$.

In the remainder of this section we indicate the long and short time limit of $\psi(t) \equiv 2 d V(t)$, calculated at fixed concentration. These long time limits can be obtained from the small $z$ expansion of the EMA equation. The details are given in appendix $A$. Three cases are distinguished, $\varepsilon>0$ (corresponding to $\left.c<c_{0}\right), \varepsilon=0$, and $\varepsilon<0$. The results are then

Case (a): Above the percolation threshold $(\varepsilon>0)$

$$
\begin{array}{ll}
\psi(t) \sim-\frac{(1-\varepsilon)}{\pi \varepsilon} t^{-2}\left[1+\frac{4}{\pi}\left(\varepsilon^{2} t\right)^{-1} \ln \frac{t}{\tau}\right], & d=2, \\
\psi(t) \sim-\frac{3 A}{8 \sqrt{\varepsilon}} \frac{(1-\varepsilon)}{\varepsilon^{3 / 2}} t^{-5 / 2}\left[1+B\left(\varepsilon^{2} t\right)^{-1}\right], & d=3,
\end{array}
$$

where $A, B$ and $\tau$ are constants defined in appendix A. 
Case (b): Below the percolation threshold $(\varepsilon<0)$

In this case $\Psi(z)$ is analytic in $z$ about $z=0$, and the small $z$ expansion (A.10) is not Laplace invertible. The specific functional dependence on $t$ therefore is not determined by this expansion, but the long time decay is of exponential order. In the next section a more explicit form will be given.

Case $(c)$ : At the percolation threshold $(\varepsilon=0)$

$$
\begin{array}{ll}
\psi(t) \sim-\frac{1}{2 \pi \sqrt{2}}[\ln (C t)]^{1 / 2} t^{-3 / 2}, & d=2, \\
\psi(t) \sim-\frac{a}{2 \sqrt{\pi}} t^{-3 / 2}+\frac{b}{\Gamma\left(-\frac{3}{4}\right)} t^{-7 / 4}, & d=3,
\end{array}
$$

where $C, a$, and $b$ are constants defined in appendix $\mathrm{A}$.

An important characteristic of the expansions away from the threshold is a divergence in the amplitude of the leading asymptotic time dependence as $\varepsilon \rightarrow 0$. The fact that the expansions are not uniform in $\varepsilon$ is clear from the first correction terms indicated in case (a). They show that the asymptotic form is approached only for times greater than $\varepsilon^{-2}$. As $\varepsilon \rightarrow 0$, this time domain shifts out to infinite times, while at long but finite times the dynamics of case (c) takes over, as will be shown in the next section.

There is a third domain for very short times, $t<1$, which is not affected by the percolation threshold, as the random walk has not yet probed the connectivity of the underlying structure. To describe this short time domain, the EMA equation (2.8) is solved as a power series in $1 / z$ using

$$
L(z)=z^{-1}-z^{-2}+z^{-3}(2 d+1) / 2 d+\cdots
$$

with the result, valid for large $z \gg 1$ :

$$
\Psi(z)=1-c-z^{-1} c(1-c) / d-z^{-2} c(1-c)\{2 d-1-(2 d-3) c\} / 2 d^{2} .
$$

The corresponding form for the VACF, $\psi(t)$, valid for $t \ll 1$ can be obtained from a term-by-term Laplace inversion of (2.15). The high frequency term, $\Psi(\infty)=1-c$, yields a delta function $(1-c) \delta(t)$. The EMA result up to order $z^{-2}$ included is exact for all concentrations. Higher order terms, however, are not exact, as a comparison with ref. [8] shows. 


\section{Crossover dynamics}

The crossover between the three types of long time dynamics can be investigated by direct numerical solution of the EMA equation, and the results of such a calculation are presented in section 4 . Ilowever, for very small $|\varepsilon|$ it is possible to extract the crossover behavior analytically from the asymptotic form of the EMA equation. Since there is a wide range of times for which each of the three types of dynamics is applicable near the threshold, we present this analysis first.

The results of section 2 indicate that the characteristic time scale separating the different domains is proportional to $\varepsilon^{-2}$. Accordingly, the EMA equation is written in terms of the scaled variables,

$$
\Psi^{*}\left(z^{*}\right) \equiv 2 \Psi(z) /|\varepsilon|(1-\varepsilon), \quad z^{*} \equiv z / \varepsilon^{2}
$$

The factor $(1-\varepsilon)$ is not relevant near the threshold, but is convenient for representing the results of the numerical evaluation of the EMA at larger positive $\varepsilon$ values in section 4 . The corresponding scaled time dependent quantities are

$$
\psi^{*}\left(t^{*}\right)=2 \psi(t) /|\varepsilon|^{3}(1-\varepsilon), \quad t^{*} \equiv \varepsilon^{2} t
$$

For small $\varepsilon$, the EMA equation takes the form

$$
\Psi^{* 2}-2 \sigma \Psi^{*}-\left[4 z^{*} /(d-1)\right] L\left(2 z^{*}|\varepsilon| / \Psi^{*}\right)=0,
$$

where $\sigma=\operatorname{sgn}(\varepsilon)$. The three different time domains of interest correspond to $\varepsilon^{-2} \ll z^{*}$ (short times), $1 \ll z^{*} \ll \varepsilon^{-2}$ (percolation dynamics), and $z^{*} \ll 1$ (long times). For sufficiently small $|\varepsilon|$, all domains can be identified clearly. In the following, we analyze eq. (3.3) in the range $z^{*} \ll \varepsilon^{-2}$, and therefore concentrate only on the crossover between long time and percolation behavior. The crossover to short time behavior, which is independent of the percolation threshold, is discussed in section 4.

The long time behavior for $\varepsilon>0$ is qualitatively different from that for $\varepsilon<0$. In the former case there is a crossover from percolation dynamics to long time tails, whereas in the latter case there is a crossover to a more rapidly decaying dynamics. However, it will appear that the percolation dynamics is the same for both cases.

The analysis is based on the assumption that $z^{*}|\varepsilon| / \Psi^{*} \ll 1$ when both $z^{*}$ and $|\varepsilon|$ are small. This assumption is verified a posteriori. Then the function $L(x)$ in eq. (3.3) can be expanded for small values of its argument using eqs. (A.1) and 
(A.2) of appendix A. Consider first the case of three dimensions. Then eq. (3.3) reduces to

$$
\Psi^{* 2}-2 \sigma \Psi^{*}-2 z^{*}\left[L_{0}-A\left(2 z^{*}|\varepsilon| / \Psi^{*}\right)^{1 / 2}\right]=0
$$

or, equivalently,

$$
\Psi^{*}\left(z^{*}\right)=\sigma+\left\{1+2 z^{*}\left[L_{0}-A\left(2 z^{*}|\varepsilon| / \Psi^{*}\right)^{1 / 2}\right]\right\}^{1 / 2} .
$$

In the percolation dynamics domain, $1 \ll z^{*} \ll \varepsilon^{-2}$, the term containing $|\varepsilon|$ can be neglected, leaving $\Psi^{*} \sim \sigma+\left[1+4 a^{2} z^{*}\right]^{1 / 2}$, with $a^{2}=L_{0} / 2$. It is easily verified that this result is consistent with the assumption that $|\varepsilon| z^{*} / \Psi^{*} \ll 1$. The corresponding time dependent function for $\varepsilon^{2} \ll t^{*} \ll 1$ is found to have the universal ( $\varepsilon$ independent) form

$$
\psi^{*}\left(t^{*}\right) \sim-(a / \sqrt{\pi})^{1 / 2} t^{*-3 / 2} \exp \left(-t^{*} / 4 a^{2}\right)
$$

This expression is equal to the second derivative of the closed form expression for the mean square displacement derived in eq. (27) of Derrida et al. [13], and is valid in the same time interval. Comparison with eq. (2.13) identifies this as the percolation dynamics with a small exponential modulation. This result applies for small $|\varepsilon|$ both above and below the threshold.

To calculate the long time behavior it is necessary to obtain the solution to eq. (3.5) for $z^{*} \ll 1$. It might appear that the term proportional to $|\varepsilon|$ could be neglected as above. However, this term gives a square root branch point for $\varepsilon>0$ which determines the leading long time dependence. It can be treated as a perturbation and (3.5) simplifies for small $z^{*}$ to

$$
\Psi^{*}\left(z^{*}\right) \sim \sigma+1+z^{*}\left[L_{0}-A\left(2 z^{*}|\varepsilon| / \Psi^{*}\right)^{1 / 2}\right] .
$$

Above the threshold $\sigma=1$ and iteration of this equation gives $\Psi^{*} \sim 2-$ $A \varepsilon^{1 / 2} z^{* 3 / 2}$, plus terms analytic at $z^{*}=0$. This implies the long time tail of eq. (2.11),

$$
\mu^{*}\left(t^{*}\right) \sim-\left(3 A \varepsilon^{1 / 2} / 4 \pi^{1 / 2}\right) t^{*-5 / 2},
$$

which is valid for $t^{*} \gg 1$. However, the amplitude is seen to be proportional to $\varepsilon^{1 / 2}$. The crossover to long time tails therefore vanishes as $\varepsilon \rightarrow 0$ in three dimensions.

Below the threshold $\sigma=-1$ and iteration of eq. (3.7) leads to $\Psi^{*} \sim$ $z^{*}\left[L_{0}-A\left(2 z^{*}|\varepsilon| / L_{0}\right)^{1 / 2}\right]$ which is regular around $z^{*}=0$. This result agrees 
with the small $z$-behavior of eq. (A.10) obtained at fixed $\varepsilon<0$, when $|\varepsilon|$ approaches zero. The associated time dependence is not uniquely determined by this expansion, but has the general form

$$
\psi^{*}\left(t^{*}\right) \sim B^{*}\left(t^{*}\right) \exp \left(-z_{0}^{*} t^{*}\right)
$$

where $t^{*}>1$. Here $z_{0}^{*}$ is the location of the singularity of $\Psi^{*}\left(z^{*}\right)$ nearest to the origin, and $B^{*}\left(t^{*}\right)$ is a function varying more slowly than the exponential. As indicated above, there are no long time tails for $\varepsilon<0$. However, the percolation tails $\sim t^{*-3 / 2}$ of eq. (3.6) are observed as intermediate behavior for $\varepsilon^{2} \ll t^{*} \ll 1$.

Next consider the case of two dimensions. Use of eq. (A.1) in eq. (3.3) yields the asymptotic EMA in two dimensions,

$$
\Psi^{*}\left(z^{*}\right)=\operatorname{sgn}(\varepsilon)+\left[1+\left(4 z^{*} / \pi\right) \ln \left(4 \Psi^{*} / z^{*}|\varepsilon|\right)\right]^{1 / 2}
$$

There is now an inherent $\varepsilon$ dependence due to the $\ln \mid \varepsilon$ contribution that cannot be removed by scaling. In the percolating domain, $z^{*} \ln \left(\Psi^{*} / z^{*}|\varepsilon|\right) \gg 1$ and eq. (3.10) reduces to $\Psi^{*} \sim\left[\left(4 z^{*} / \pi\right) \ln \left(4 \Psi^{*} / z^{*}|\varepsilon|\right)\right]^{1 / 2}$. The solution to this equation is given by eq. (A.8) of appendix $A$,

$$
\Psi^{*}\left(z^{*}\right) \sim\left[\left(2 z^{*} / \pi\right) \ln \left(32 / \pi z^{*} \varepsilon^{2}\right)\right]^{1 / 2}
$$

The corresponding time dependence is the percolation dynamics of eq. (2.12),

$$
\psi^{*}\left(t^{*}\right) \sim-(\pi \sqrt{2})^{-1}\left[\ln \left(C t^{*} / \varepsilon^{2}\right)\right]^{1 / 2} t^{*-3 / 2} .
$$

For intermediate times where $1<z^{*} \ln \left(\Psi^{*} /|\varepsilon|\right)$ the quantity $\ln \left(\Psi^{*} / z^{*}\right)$ changes only very slowly and the time dependence is given qualitatively by (3.12) with an exponential modulation similar to (3.6). Again, these results hold both above and below the threshold.

The long time dynamics is obtained from the $z^{*} \ll 1$ behavior, for which $z^{*} \ln \left(\Psi^{*} / z^{*}|\varepsilon|\right) \ll 1$. Then eq. (3.10) reduces to

$$
\Psi^{*}\left(z^{*}\right) \sim \sigma+1+\left(2 z^{*} / \pi\right) \ln \left(4 \Psi^{*} / z^{*}|\varepsilon|\right) .
$$

For $\varepsilon>0, \sigma=1$ and iteration leads to the solution

$$
\Psi^{*}\left(z^{*}\right) \sim 2\left[1-\left(z^{*} / \pi\right) \ln \left(z^{*}|\varepsilon| / 8\right)\right] .
$$


This corresponds to the long time tails of eq. (2.10) in two dimensions as $\varepsilon \rightarrow 0$,

$$
\psi^{*}\left(t^{*}\right) \sim-(2 / \pi) t^{*-2} .
$$

In contrast to the three-dimensional case, the amplitude of this tail is independent of $\varepsilon$, and thus competitive with the percolation dynamics. Below the threshold, $\sigma=-1$ and eq. (3.13) yields $\psi^{*} \sim\left(2 z^{*} / \pi\right) \ln \left(4 \Psi^{*} / z^{*}|\varepsilon|\right)$. This has the solution given by eqs. (A.10) and (A.12) of appendix A,

$$
\Psi^{*}\left(z^{*}\right) \sim\left(2 z^{*} / \pi\right) \ln (8 / \pi|\varepsilon|),
$$

which is regular around $z^{*}=0$. The associated time dependence is therefore similar to eq. (3.9) for the three-dimensional case.

In summary, eqs. (3.5) and (3.10) are the asymptotic forms for the EMA equation at small $\varepsilon$ in two and three dimensions, respectively. They describe the complete crossover between long time and percolation dynamics both above and below the threshold, as indicated by the above analysis.

\section{Numerical results}

A more quantitative description of the crossover behavior between these different types of dynamics near the threshold is easily obtained from numerical solution to eqs. (3.5) and (3.10). More generally, for larger $\varepsilon$ values it is necessary to solve the full EMA equation (2.8). To illustrate the results, we consider in detail the most interesting case of two dimensions for a wide range of concentrations above and below the threshold. For real $\omega^{*}$, the real and imaginary parts of $\Psi^{*}\left(z^{*}=i \omega^{*}\right)$ are proportional to the Fourier cosine and sine transforms of $\psi^{*}(t)$. The corresponding time correlation function $\psi^{*}\left(t^{*}\right)$ is then obtained by Fourier inversion. To help interpret the results in the frequency representation note when $\Psi^{*}\left(z^{*}\right)$ is regular around $z^{*}=0$, then $\operatorname{Re}\left[\Psi^{*}\left(\mathrm{i} \omega^{*}\right)-\Psi^{*}(0)\right] \sim \omega^{*^{2}}$ and $\operatorname{Im} \Psi\left(\mathrm{i} \omega^{*}\right) \sim \omega^{*}$ for small $\omega^{*}$. On a $\log -\log$ plot these curves would have slopes 2 and 1, respectively. Furthermore, in the asymptotically large frequency domain, eq. (2.15) implies that the $\log -\log$ plots of $\operatorname{Re} \Psi^{*}$ and $\operatorname{Im} \Psi^{*}$ would have slopes 0 and -1 .

Consider first the case $\varepsilon>0$ far above the threshold. Figs. 1a and 1b show, respectively, the real and imaginary parts of $\Psi^{*}\left(i \omega^{*}\right)$ for $c=0.1,0.2,0.3,0.4$, 0.45 and 0.49 . Eq. (3.14) gives the asymptotic behavior $\left[\operatorname{Re} \Psi^{*}-2\right] \sim \omega^{*}$ and $\operatorname{Im} \Psi^{*} \sim-\omega^{*} \ln \left(\varepsilon \omega^{*}\right)$ for $\omega^{*} \ll 1$. This characterizes the long time tails and appears in figs. 1a, b as the domain with slope 1 . In this domain $\operatorname{Re} \Psi^{*}$ is 
G.A. van Velzen et al. / Crossover dynamics in bond disordered lattices
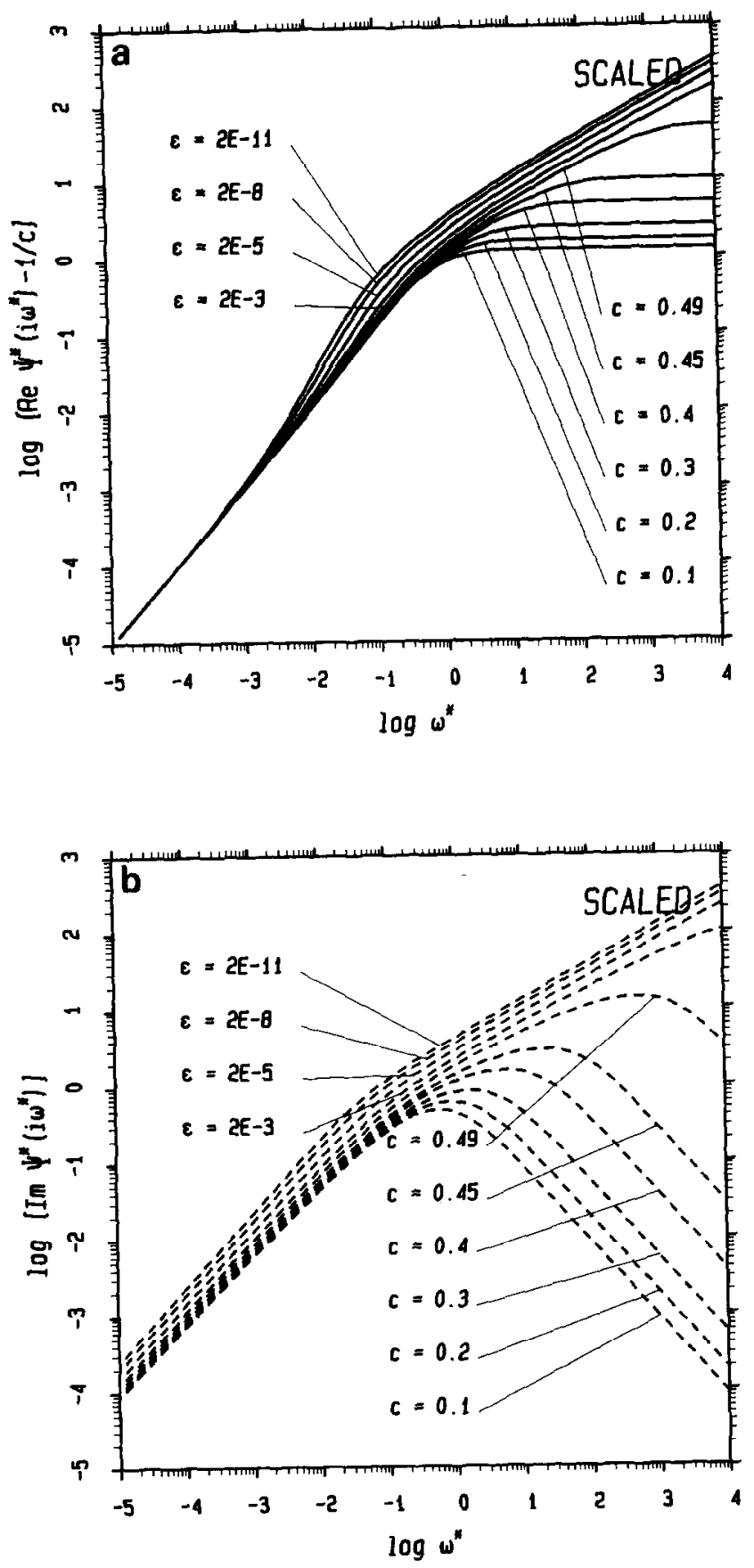

Fig. 1. $\Psi^{*}\left(z^{*}=\mathrm{i} \omega^{*}\right)$ above threshold. Real (a) and imaginary (b) parts of $\left[\Psi^{*}\left(\mathrm{i} \omega^{*}\right)-\Psi^{*}(0)\right]$ are shown as a function of $\omega^{*}$ for several values of $c$ and $\varepsilon \equiv\left(c_{0}-c\right) / c_{0} .\left({ }^{10} \log\right.$ used in all figures.) 
universal, while $\operatorname{Im} \Psi^{*}$ shows a weak logarithmic dependence on $\varepsilon$. For large $\omega^{*}, \Psi^{*}$ is regular and, as noted above, the real and imaginary parts should approach $\log -\log$ slopes of 0 and -1 , respectively. The crossover to this high frequency limit is seen to occur for $\omega^{*}>\varepsilon^{-2} \sim 1-10$ for these values of $\varepsilon$. The shift of this domain to larger frequencies, as $\varepsilon$ decreases, marks the formation of a new intermediate frequency domain which is the percolation dynamics. For a large $\varepsilon$ there is a simple short time dynamics $\left(\omega^{*}>\varepsilon^{-2}\right)$ and long time tails $\left(\omega^{*}<1\right)$.

Next we consider in figs. $1 \mathrm{a}, \mathrm{b}$ the same quantities, still above threshold, but for much smaller $\varepsilon\left(\varepsilon=2 \times 10^{-3}, 2 \times 10^{-5}, 2 \times 10^{-8}\right.$ and $\left.2 \times 10^{-11}\right)$, with $\varepsilon=$ $\left(c_{0}-c\right) / c_{0}=1-2 c$. The domain of long time tails is unchanged, but the short time dynamics occurs only for larger frequencies than those shown. Instead, the region $1<\omega^{*}<\varepsilon^{-2}$ is characterized by eq. (3.11), representing the percolation dynamics, for which both $\operatorname{Re} \Psi^{*}$ and $\operatorname{Im} \Psi^{*}$ have slopes $\cong \frac{1}{2}$ on the $\log -\log$ plot. Again, there is a weak logarithmic dependence on $\varepsilon$ in this domain, reflecting the breakdown of scaling in two dimensions. The crossover between long time tails and percolation dynamics is seen to occur for $10^{-2}<$ $\omega^{*}<10^{0}$.

Figs. 2a, b show the same data for concentrations below threshold. It was shown in section 3 that the percolation dynamics is the same just above and just below the threshold; so the domain $1<\omega^{*}<\varepsilon^{-2}$ is essentially unchanged from fig. 1. However, below threshold, there are no long time tails. Instead, $\Psi^{*}$ is regular for $\omega^{*}<1$, and the real and imaginary parts have slopes 2 and 1 , respectively, on the $\log -\log$ plots. This crossover between percolation dynamics and the regular low frequency form (exponential time decay) is clearly shown in fig. 2 . Here the low frequency behavior is not universal as in fig. 1a.

The larger values of $c(c \geqslant 0.51)$ shown in fig. 2 indicate the approach to short time dynamics at the highest frequencies. Since this corresponds to $\omega^{*}>|\varepsilon|^{-2}$, the domain of short time dynamics dominates the percolation dynamics far above the threshold, just as it does far below. The percolation dynamics is evident for $|\varepsilon|=2 \times 10^{-2}, 2 \times 10^{-5}, 2 \times 10^{-8}$ and $2 \times 10^{-11}$ and $\omega^{*} \geq 0.1$. The domain $\omega^{*} \ll 0.1$ shows regular small frequency behavior (A.10) where the coefficients have logarithmic $|\varepsilon|$-dependence as given through (A.13).

We proceed with the calculations of the time dependence of the velocity autocorrelation function (VACF) by Fourier inversion of the numerically obtained solution to the EMA equation (2.9). We were able to obtain results over a time interval $10^{-2}<t^{*}<1000$. Figs. 3a and 4a show $\psi^{*}\left(t^{*}\right)$ vs $t^{*}$ above and below threshold, respectively. Figs. $3 \mathrm{~b}$ and $4 \mathrm{~b}$ show the unscaled quantities $\psi(t)$ vs $t$ for the same $\varepsilon$-values. There is no percolation dynamics for $c \leqslant 0.45$ and $c \geqslant 0.55$; the scaled and unscaled graphs are qualitatively the same. They 

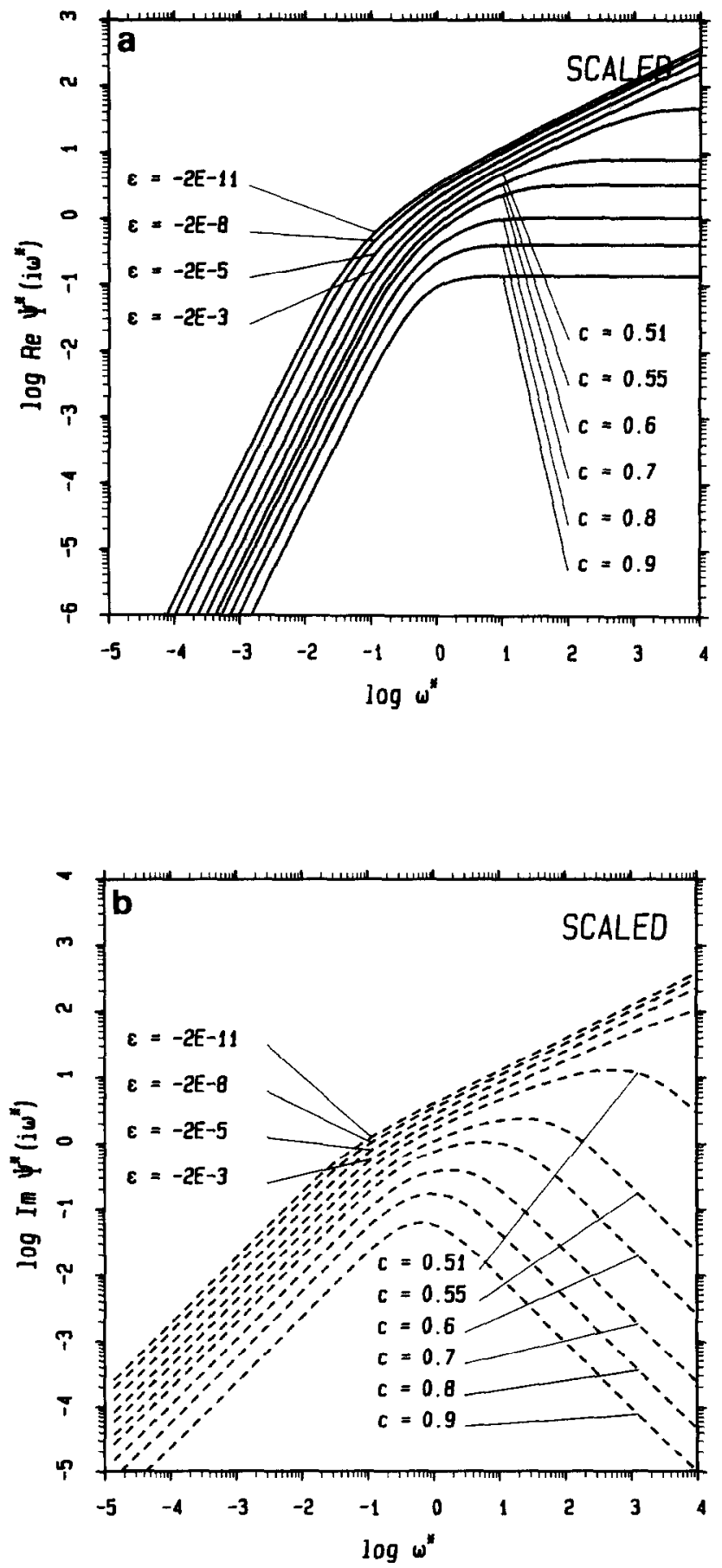

Fig. 2. Same as figs. 1a and b except below threshold. 

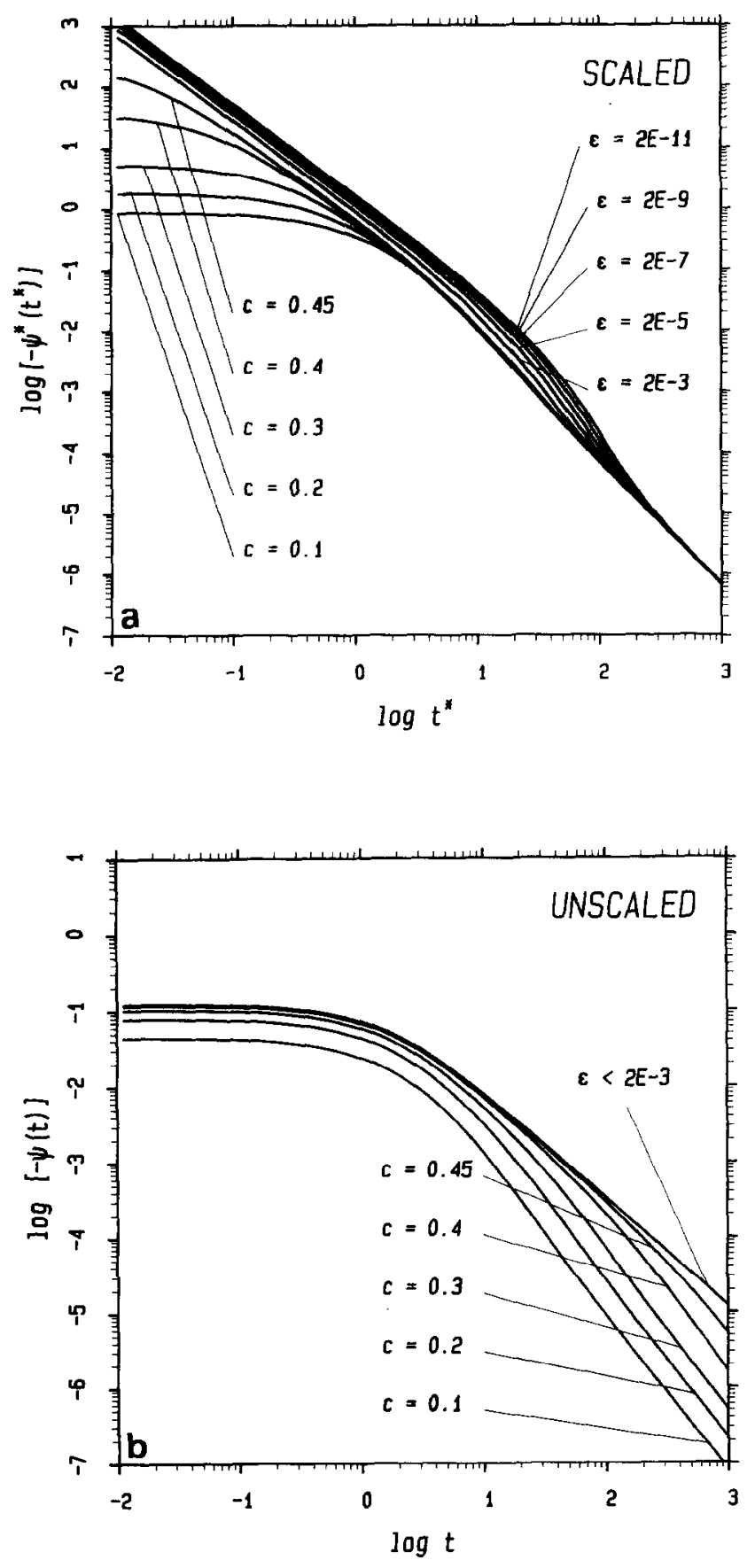

Fig. 3. (a) Scaled VACF $\psi^{*}\left(t^{*}\right)$, above threshold. (b) Unscaled VACF $\psi(t)$, above threshold. 

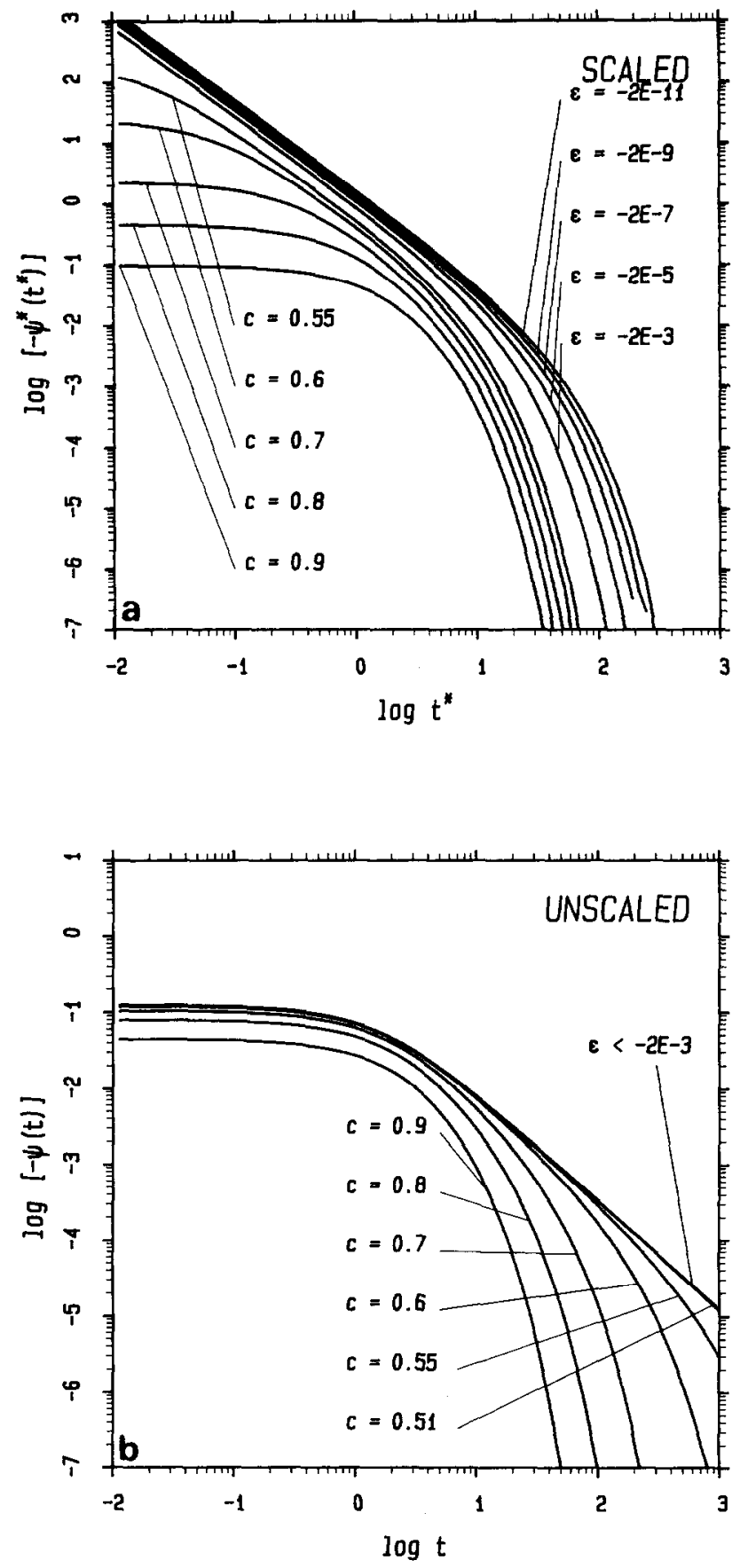

Fig. 4. Same as figs. 3a and $b$, except below threshold. 
show crossover from short time dynamics to long time tails in fig. 3. Below threshold (fig. 4) the short time dynamics crosses over to exponential decay, as expected.

Close to the threshold a percolation domain develops for $\varepsilon^{2}<t^{*}<1$ or $1<t<\varepsilon^{-2}$. The percolation dynamics shows a weak logarithmic $\varepsilon$-dependence (see eq. (3.12)) that also exists in the frequency domain. This occurs for small $|\varepsilon|$ and $t^{*}<10$ (figs. 3a and 4a). The long time tail, derived in eq. (2.10), is essentially given by the lower envelope of fig. $3 \mathrm{a}$ for $t^{*} \geqslant 40$. Evidently the final crossover to long time tails at small $\varepsilon$ occurs only at $t^{*} \approx 100$.

Finally, we consider the exponential decay of the VACF below the percolation threshold or, equivalently, the crossover times from critical dynamics to exponential decay, as a function of the concentration $c$. A numerical estimate of the characteristic decay time is obtained from the ratio of the coefficients in the small $z$-expansion (A.10) of $\Psi(z)$, namely $\tau(\varepsilon) \sim\left|\alpha_{2}(\varepsilon) / \alpha_{1}(\varepsilon)\right|$. The relevant data are plotted in fig. 5. They show that the dominant $\varepsilon$-dependence is given by $\alpha_{1} \sim|\varepsilon|^{-1}$ and $\alpha_{2} \sim|\varepsilon|^{-3}$, as derived in (A.13).

An alternative estimate, $\tau_{1}(\varepsilon)$, for the exponential decay time can be obtained from the data in fig. 4a by assuming that the VACF is qualitatively

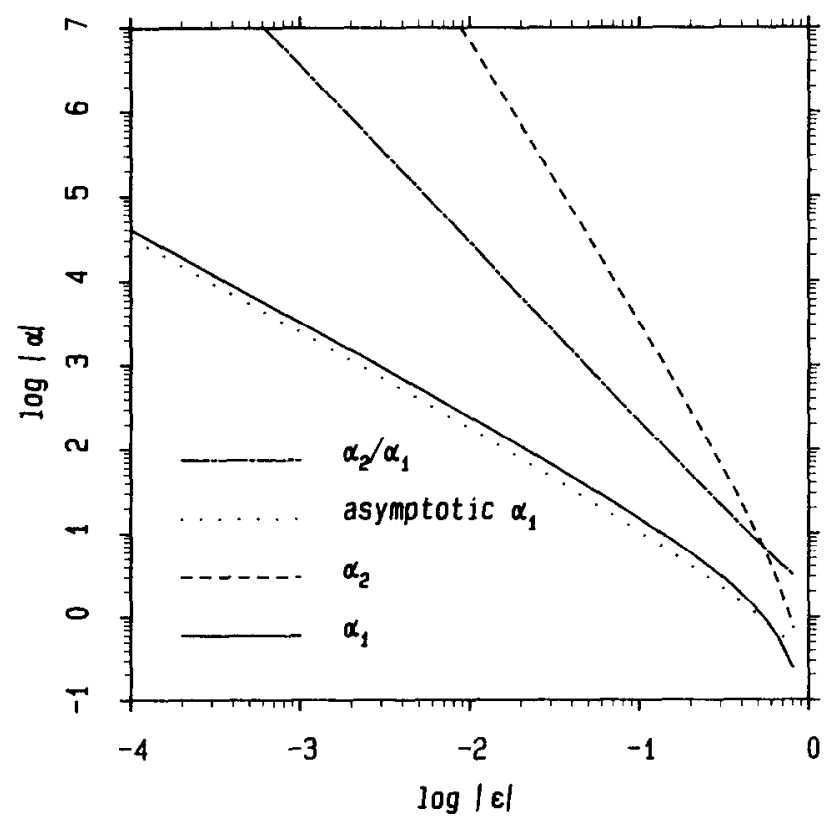

Fig. 5. Coefficients, $\alpha_{1}(\varepsilon)$ and $\alpha_{2}(\varepsilon)$, in small $z$ expansion of $\Psi(z)$ (see eq. (A.10)). Also shown is estimate of decay time $\tau(\varepsilon) \equiv\left|\alpha_{2}(\varepsilon) / \alpha_{1}(\varepsilon)\right|$. The asymptotic form of $\alpha_{1}(\varepsilon)$ from eq. (A.12) is represented by the dotted line. 
given by $\Psi(t) \sim \exp \left(-t / \tau_{1}(\varepsilon)\right)$. The resulting $\tau_{1}(\varepsilon)$-values are proportional to $\tau(\varepsilon)$ above. The constant of proportionality varies from 1.5 at $c=0.9$ to $\approx 3.0$ at the small $\varepsilon$-values in fig. 4a.

\section{Discussion}

We summarize the EMA results in terms of scaling forms and corrections to scaling for dimensionality $d>2$ (see eq. (3.13) of ref. [2]). The essential input in the EMA equation (2.8) is the dominant small $z$ behavior and the dominant small $z$ singularity, given in eq. (A.2). For $d=2$ both types of terms coincide. From eq. (3.5) we obtain the scaling form for small $|\varepsilon|$ with $d>2$ :

$$
\begin{aligned}
& \Psi(z, \varepsilon)=\frac{1}{2}|\varepsilon| \phi_{0}\left(z^{*}\right), \\
& \phi_{0}\left(z^{*}\right)=\sigma+\left[1+4 a^{2} z^{*}\right]^{1 / 2}
\end{aligned}
$$

where again $\sigma=\operatorname{sgn}(\varepsilon), a^{2}=L_{0} /(d-1)$, and $z^{*}=z / \varepsilon^{2}$. The scaling function $\phi_{0}(x)$ is regular and describes the percolation dynamics. To obtain the corresponding function in the time representation, it is convenient to calculate first the mean square displacement by inverting the Laplace transform of $\Psi(z, \varepsilon) /$ $z^{2}$. This yields the scaling form of eq. (1.1) for $d>2$ [13]:

$$
\left\langle r^{2}\right\rangle(t, \varepsilon)=t|\varepsilon|^{x} F\left(|\varepsilon|^{y} t\right)
$$

with $x=1, y=2$, and $F\left(|\varepsilon|^{y} t\right)=F\left(t^{*}\right)$ given by

$$
\begin{aligned}
F\left(t^{*}\right)= & \frac{1}{2}\left\{(\sigma+1)+\left(2 a^{2} / t^{*}\right)\right. \\
& \left.-8 a^{3} t^{*-3 / 2} \mathrm{e}^{-t^{*} / 4 a^{2}} \int_{0}^{\infty} \mathrm{d} s \mathrm{e}^{-s}(1-s)\left[\pi\left(1+4 a^{2} s / t^{*}\right)\right]^{-1 / 2}\right\} .
\end{aligned}
$$

It has been written in this form to show explicitly that the last term inside the brackets is decreasing exponentially fast. We further note that the above results and eq. (A.9) imply that the mean square displacement at threshold $\sim t^{1 / 2} \sim$ $t^{2 / d_{w}^{\prime}}$, where $d_{w}^{\prime}=4$ is the fractal dimension of the random walk. This is the same exponent as obtained for the mean square displacement of a random walk on a one dimensional random walk (see eq. (8.18) of ref. [1]). The time dependent VACF is obtained from the mean square displacement by differen- 
tiating twice (see eq. (2.3)) to obtain the asymptotic form for $d>2$,

$$
\psi(t, \varepsilon) \sim \frac{1}{2}|\varepsilon|^{3} F_{0}\left(t^{*}\right),
$$

with the scaling function

$$
F_{0}\left(t^{*}\right)=\psi^{*}\left(t^{*}\right)=-(a / \sqrt{\pi}) t^{*-3 / 2} \mathrm{e}^{-t^{*} / 4 a^{2}} .
$$

This is the percolation dynamics that dominates at the threshold, or more precisely, for $|\varepsilon|^{2} \ll t^{*}<1$.

Next, we include the singular terms $z^{-1+d / 2}$ in $L(z)$ which are responsible for the long time dynamics away from the threshold, and obtain from eq. (2.8) corrections to scaling:

$$
\begin{aligned}
& \Psi(z, \varepsilon)=\frac{1}{2}\left\{|\varepsilon| \phi_{0}\left(z^{*}\right)+|\varepsilon|^{d / 2} \phi_{1}\left(z^{*}\right)\right\}, \\
& \phi_{1}(x)=A(2 x)^{d / 2}\left[\phi_{0}(x)\right]^{1-d / 2}\left\{(d-1)\left(\phi_{0}(x)-\sigma\right)\right\}^{-1} .
\end{aligned}
$$

Again using eq. (2.3), the corresponding time correlation function is found to be, for $d>2$,

$$
\psi(t, \varepsilon)=\frac{1}{2}\left[\varepsilon^{3} F_{0}\left(t^{*}\right)+\varepsilon^{2+d / 2} F_{1}\left(t^{*}\right)\right] .
$$

These results generalize eq. (5.4) to the entire time domain $t^{*} \gg|\varepsilon|^{2}$. The qualitative behavior of $F_{1}\left(t^{*}\right)$ follows from (5.2), (5.6) and (5.7) as

$$
F_{1}\left(t^{*}\right) \sim \begin{cases}t^{*-1-d / 2} & \left(t^{*} \gg 1, \varepsilon>0\right), \\ t^{*-1-d / 4} & \left(\varepsilon^{2} \ll t^{*} \ll 1\right), \\ \cdot \exp \left(-t^{*}\right) " & \left(t^{*} \gg 1, \quad \varepsilon<0\right),\end{cases}
$$

where "exp $\left(-t^{*}\right)$ " indicates that the long time behavior is of exponential type. The coefficients of all algebraic terms for $d=3$ are given in section 3 and can be calculated simply for general $d$. In the limit $\varepsilon=0$ these equations yield the percolation dynamics $\psi(t, \varepsilon) \sim-t^{-3 / 2}+t^{-1-d / 4}$. The last term represents the subleading critical tail for $d<6$.

The results in eqs. (5.8) and (5.9) above threshold represent also the long time tails, and illustrate the complex relationship between critical and asymptotic dynamics. For any fixed $\varepsilon$ above the threshold there exists a time $t_{1}^{*}(\varepsilon)$ such that for $t^{*}>t_{1}^{*}$ the term $F_{1}$ in eq. (5.8) with an algebraic tail dominates $F_{0}$ with an exponential decay. To obtain an estimate for $t_{1}^{*}(\varepsilon)$ in three dimensions we equate both terms in (5.6), yielding the transcendental equation $t_{1}^{*}=$ 
$3 A(\varepsilon / a \sqrt{\pi})^{1 / 2} \exp \left(t_{1}^{*} / 4 a^{2}\right)$. The solution is approximately described by $t_{1}^{*}=$ $7-\frac{3}{4} \ln (\varepsilon)$ over the interval $10^{-8}<\varepsilon<10^{-2}$. Unfortunately, we have no estimate for $t_{1}^{*}$ in the two-dimensional case.

The equation corresponding to (5.8) for two-dimensional systems exhibits an additional complication, where the correction to scaling is of the same order in $\varepsilon$ as the leading term. The above analysis for $d>2$ breaks down because the dominant singularity and dominant small $z$ behavior of $L(z)$ coincide. The detailed analysis and numerical solution of the EMA equation for $d=2$ was presented in sections 3 and 4 .

Below the threshold, eq. (5.8) shows a crossover from percolation dynamics with a critical tail $t^{-3 / 2}$ to an exponential decay on a time scale determined by $\tau(\varepsilon) \sim\left|\alpha_{2} / \alpha_{1}\right|$ with coefficients determined from eqs. (A.10)-(A.13). The mean square displacement is obtained from $\Psi(z, \varepsilon) / z^{2}$ in eq. (A.10) by Laplace inversion and is bounded by a constant for long times, $\left\langle r^{2}\right\rangle(t, \varepsilon) \approx \alpha_{1}(\varepsilon) / 2 d \equiv$ $R_{\infty}^{2}$. The behavior of these quantities near threshold is given by eq. (A.13) and is related to the critical exponents in eq. (1.1) as $R_{\infty}^{2} \sim|\varepsilon|^{x-y}$ and $\tau(\varepsilon) \sim|\varepsilon|^{-y}$. The EMA values of these exponents are very different from mean field values (see table I).

We also consider the average density, $N(E)$, of eigenstates for the master equation (2.1) (i.e., $\Sigma_{m} L_{n m} g_{m}=E g_{n}$ ). This density is related to the single site Green's function, $\hat{P}_{0}(-E+\mathrm{i} 0)$, by (see eq. (2.8) of ref. [2])

$$
N(E)=-\frac{1}{\pi} \operatorname{Im} \hat{P}_{0}(-E+\mathrm{i} 0) .
$$

The EMA for $\hat{P}_{0}(z)$ is obtained from eq. (2.7) by replacing $\omega(q)$ by $\omega(q) \Psi(z)$ (see eq. (6.81) of ref. [1]), so that

$$
\hat{P}_{0}(z)=L(z / \Psi(z)) / \Psi(z) .
$$

This yields for the spectral density above or at threshold at small $E$-values a phonon or fracton type behavior, respectively,

$$
N(E) \sim \begin{cases}\pi^{-1} & (\varepsilon>0, \quad d=2), \\ E^{-1 / 2}[-\ln (E)]^{1 / 2} & (\varepsilon=0),\end{cases}
$$

and

$$
N(E) \sim \begin{cases}E^{-1+d / 2} & (\varepsilon>0, \quad d>2), \\ E^{-1 / 2} \sim E^{-1+d_{s} / 2} & (\varepsilon=0)\end{cases}
$$

with a spectral dimension $d_{\mathrm{s}}=1[13]$. 
Quantitative comparisons of EMA results with computer simulations have been made only for the mean square displacement for $d=1,2$ and 3 (see figs. 6.4-6.7 of ref. [13]) and show good agreement at short and long times. The leading behavior of the VACF follows from these data only after taking derivatives twice, and no quantitative comparison has been made in the literature. Preliminary results of Brey et al. [17] show that there is quantitative agreement of the EMA predictions for the VACF with their simulation results for the 2D bond percolation model both above $(c=0.2$ and $c=0.3)$ and below $(c=0.7)$ the threshold. The agreement is very good up to $t \sim 40$. Small deviations start to appear as $t$ increases to 100 .

Far below threshold Vicsek [21] has performed computer simulations for the two dimensional bond problem to determine $\operatorname{Re} \Psi(\mathrm{i} \omega)$ and $\operatorname{Im} \psi(\mathrm{i} \omega)$ at small frequencies, where $\Psi(z)$ in eq. (A.10) is regular and the corresponding exponential time decay is predominant. His results are in complete agreement with the slopes in figs. 2a, b. To make a comparison of his data with the coefficients in eq. (A.10) we refer to fig. 5, containing $\log -\log$ plots of $R_{\infty}^{2}(\varepsilon) \sim \alpha_{1}(\varepsilon) \sim|\varepsilon|^{-u}$ with $u=y-x$ and $\tau(\varepsilon) \sim \alpha_{2}(\varepsilon) / \alpha_{1}(\varepsilon) \sim|\varepsilon|^{-v}$ versus $|\varepsilon|$ over the whole density interval. Vicsek determines effective exponents over an $|\varepsilon|$ interval $0.09-0.8(d=2)$ and 1.3-0.07 $(d=3)$. His results are: $u_{\mathrm{eff}} \simeq$ $2.4(d=2)$ and $1.2(d=3)$ as compared to the results from scaling in table I, $u_{\text {scal }} \simeq 2.5(d=2)$ and $1.3(d=3)$, and the EMA value $u_{\mathrm{EMA}} \simeq 1$. Similarly, $v_{\text {eff }} \simeq 3.0(d=2)$ and $2.1(d=3)$ as compared to scaling, $v_{\text {scal }} \simeq 3.8(d=2)$ and $3.4(d=3)$, and to the EMA value $v_{\mathrm{EMA}}=2$. His $u$-exponents are in fair agreement with the scaling predictions, although his $v$-exponents are not. Agreement with scaling is not expected over the complete range of $\varepsilon$-values since rather large $\varepsilon$-values are included. Nevertheless, fig. 5 shows that within the EMA the power law dependence of $R_{\infty}(\varepsilon)$ and $\tau(\varepsilon)$ holds for all $\varepsilon \leqslant 0.5$. In three dimensions $u_{\text {eff }}$ and $v_{\text {eff }}$ are close to the values obtained from the EMA.

To summarize, we have demonstrated to what extent the EMA gives a quantitative description of the behavior of the VACF over the whole density interval in a bond percolation model, which is a type of lattice Lorentz gas. The model provides a qualitative explanation of the long time tails observed by Alder and Alley in the two-dimensional Lorentz gas of overlapping scatterers over the density interval $c=0.02$ up to $c=0.74$ where the percolation threshold is $c_{0} \simeq 0.37$.

It is likely that the concentration dependence of the exponent for the tails that they obtain is really due to the superposition of the long time tails and the percolation dynamics. This can be seen from fig. $3 \mathrm{~b}$ and $4 \mathrm{~b}$. If it is assumed that a power law applies in the domain near $t \sim 100$ (along computer simulation time) it is seen in fig. $3 b$ that the effective exponent (slope) decreases from 2 to 1.5 as $c$ increases from 0.1 to 0.5 . As $c$ is increased further above 0.5 , fig. $4 \mathrm{~b}$ 
shows the effective exponent increases. This in indeed the qualitative behavior observed by Alder and Alley for the Lorentz gas.

For the model described here the amplitudes of the long time tails actually diverge at the threshold, indicating that it is not the physicaly relevant dynamics near threshold. This divergence of the amplitudes for the long time tails has been predicted for fluids by Keyes [22] on the basis of a scaling law like eq. (5.4) and an ansatz that the percolation dynamics and long time tails agree over some finite time interval. His basic idea is to equate the two forms of dynamics at some intermediate time, $t_{0}$. In three dimensions, equating the forms in eqs. (2.11) and (2.13) gives an amplitude for the long time tail relative to that for the percolation dynamics, $A / A_{p}=t_{0}$. Since $A_{p}$ is independent of $\varepsilon$ and the time scales as $\varepsilon^{y}$, the amplitude $A$ should diverge as $\varepsilon^{y}$. This argument fails for the case considered here because $y=-2$, while the explicit form of eq. (2.11) shows that $A$ diverges as $\varepsilon^{-3 / 2}$. Since the scaling does hold, the failure of the method lies in the assumption that there exists a $t_{0}$ for which both forms of dynamics apply.

\section{Acknowledgements}

This research was supported in part by National Science Foundation grant CHE-8411932. The work of G.A.v.V. is part of a research program of the Stichting voor Fundamenteel Onderzoek der Materie (FOM), which is financially supported by the Nederlandse Organisatie voor Zuiver-Wetershappelijk Onderzoek (ZWO). Two of us (M.H.E and G.A.v.V.) thank the Physics Department of the University of Florida for its hospitality during a stay in which most of this research was carried out.

\section{Appendix A}

\section{Long time limits}

The long time limits of section 2 are calculated from the small $z$ dependence of $\Psi$. This is determined from eq. (2.9) and the asymptotic form of $L(z)$ [9],

$$
L(z) \sim \begin{cases}\frac{1}{\pi}\left(1-\frac{z}{2}\right) \ln \frac{8}{z}+\frac{z}{2 \pi} & (d=2), \\ L_{0}+z L_{1}-A z^{-1+d / 2}\left(1-\frac{d}{4} z\right) & (d>2),\end{cases}
$$

where $L_{0} \equiv L(0)$ and $A=\pi(d / 2 \pi)^{1+d / 2} \Gamma(-d / 2)$, where $L(0) \sim 1.51638$ for 
$d=3$. The explicit value of $L_{1}$ is not needed. The term containing $A$ represents the dominant small $z$ singularity for general $d$. Explicit results are given for $d=2$ and $d=3$.

Consider first the case of $\varepsilon>0$. For three dimensions $\Psi(z)$ can be expanded in powers of $z^{1 / 2}$,

$$
\Psi(z)=a_{0}+a_{1} z^{1 / 2}+a_{2} z+a_{3} z^{3 / 2}+a_{4} z^{2}+a_{5} z^{5 / 2}+\cdots, \quad d=3 .
$$

Substitution of eqs. (A.2) and (A.4) into the EMA equation (2.8) determines the coefficients to be

$$
\begin{aligned}
& a_{0}=\varepsilon, \quad a_{1}=0, \quad a_{2}=(1-\varepsilon) L_{0} / 2 \varepsilon, \\
& a_{3}=-(1-\varepsilon) A / 2 \varepsilon^{3 / 2}, \quad a_{4}=-(1-\varepsilon)\left(4 \varepsilon^{3}\right)^{-1}\left[L_{0}^{2}-2 \varepsilon L_{1}\right], \\
& a_{5}=(1-\varepsilon)\left(8 \varepsilon^{7 / 2}\right)^{-1} A\left[L_{0}(5-\varepsilon)+3 \varepsilon\right] .
\end{aligned}
$$

The two-dimensional case is somewhat more complicated due to the logarithmic terms in eq. (A.1). The expansion now has the form

$$
\begin{aligned}
& \Psi(z)=b_{0}+b_{1} z \ln (z / 8 \varepsilon)+b_{2}[z \ln (z / 8 \varepsilon)]^{2}+b_{3} z^{2} \ln (z / 8 \varepsilon)+b_{4} z^{2}+\cdots, \\
& \quad d=2
\end{aligned}
$$

with

$$
\begin{aligned}
& b_{0}=\varepsilon, \quad b_{1}=-(1-\varepsilon) / \pi \varepsilon, \quad b_{2}=b_{1} / \varepsilon^{2} \pi, \\
& b_{3}=-\left(1+2 b_{1}\right) b_{1} / 2 \varepsilon, \quad b_{4}=-b_{1} / 2 \varepsilon .
\end{aligned}
$$

Next consider $\varepsilon=0$. Then eq. (2.8) simplifies to

$$
\Psi^{2}=(1-\Psi) z L(z / \Psi) /(d-1)
$$

and use of eqs. (A.1) and (A.2) gives the results

$$
\Psi(z) \sim[(z / 2 \pi) \ln (32 / \pi z)]^{1 / 2}, \quad d=2,
$$

where the terms neglected are of relative order $(\ln |\ln z|) / \ln z$, and

$$
\Psi(z) \sim a z^{1 / 2}+b z^{3 / 4}, \quad d=3,
$$

with $a^{2}=L_{0} / 2$ and $b=-(A / 4) a^{-3 / 2}$. 
Finally, for $\varepsilon>0$, eq. (2.9) gives $\Psi(0)=0$ and the expansion is

$$
\Psi(z)=\alpha_{1} z+\alpha_{2} z^{2}+\cdots .
$$

Substitution of (A.10) into cq. (2.8) gives

$$
\begin{aligned}
& \varepsilon(d-1) \alpha_{1}+L\left(1 / \alpha_{1}\right)=0, \\
& \alpha_{2}=-(d-1)(1+|\varepsilon|) \alpha_{1}^{4}\left[(d-1)|\varepsilon| \alpha_{1}^{2}+L^{\prime}\left(1 / \alpha_{1}\right)\right]^{-1} .
\end{aligned}
$$

Here $L^{\prime}$ denotes the derivative of $L$ with respect to its argument. Eq. (A.11) is a transcendental equation for $\alpha_{1}$. Near $\varepsilon=0, \alpha_{1}$ behaves as

$$
\alpha_{1} \sim \begin{cases}(\pi|\varepsilon|)^{-1} \ln (8 / \pi|\varepsilon|), & d=2, \\ a^{2}|\varepsilon|^{-1}, & d=3,\end{cases}
$$

and $\alpha_{2} \sim-\alpha_{1}^{2} /|\varepsilon|$. These results for the small $z$ dependence of $\Psi(z)$ agree with those of Odagaki et al. [10], and extend them to the next leading order for the case $\varepsilon>0$. Near $c=1$, eq. (A.11) can be solved as a power series in $p=1-c$, using (2.14) and $\varepsilon(d-1)=d p-1$. The result is

$$
\alpha_{1}=d p+d\left(d+\frac{1}{2}\right) p^{2}+\cdots, \quad \alpha_{2}=-d^{2} p+\cdots .
$$

The long time dependence of $\psi(t)$ for $\varepsilon \geqq 0$ is obtained using the Tauberian theorems of table II. For $\varepsilon>0$ they give

$$
\psi(t) \sim \begin{cases}b_{1} t^{-2}\left[1+\left(4 / \pi \varepsilon^{2}\right) t^{-1} \ln (t / \tau)\right], & d=2, \\ \left(a_{3} / \Gamma\left(-\frac{3}{2}\right)\right) t^{-5 / 2}\left[1+B\left(\varepsilon^{2} t\right)^{-1}\right], & d=3 .\end{cases}
$$

Table II

Tauberian theorems.

\begin{tabular}{ll}
\hline$(\mathscr{L} f)(z) \quad(z \rightarrow 0)$ & $f(t) \quad(t \rightarrow \infty)$ \\
\hline$\Gamma(-\alpha) z^{\alpha}$ & $t^{-1-\alpha}$ \\
$z^{n} \ln (z)$ & $(-1)^{n+1} n ! t^{-1-n}$ \\
$z^{n}(\ln (z))^{2}$ & $(-1)^{n} 2 n ![\ln (t)-\psi(n+1)] t^{-1-n}$ \\
$\Gamma(-\alpha) z^{\alpha} \ln (1 / z)$ & {$[\ln (t)-\psi(-\alpha)] t^{-1-\alpha}$} \\
$\Gamma(-\alpha) z^{\alpha}(\ln (C / z)]^{\beta}$ & {$[\ln (t)-\psi(-\alpha)]^{\beta} t^{-1-\alpha}\left\{1+\operatorname{order}\left(\ln ^{2}(t)\right\}\right.$} \\
$\alpha \neq 0,1,2, \ldots$ & $\psi(x)=\mathrm{d} \ln (\Gamma(x)) / \mathrm{d} x \quad$ (ref. [3]) \\
\hline
\end{tabular}


Here $B \equiv-5 a_{5} \varepsilon^{2} / 2 a_{3}=\frac{5}{8}\left[L_{0}(5-\varepsilon)+3 \varepsilon\right]$ and $\tau=(8 \varepsilon)^{-1} \exp [-\varepsilon(\pi+2) / 4+$ $2-\gamma]$, where $\gamma$ is Euler's constant. Similarly, for $\varepsilon=0$ they lead to

$$
\psi(t) \sim \begin{cases}-\frac{1}{2 \pi \sqrt{2}}[\ln (C t)]^{1 / 2} t^{-3 / 2}, & d=2, \\ \left(a / \Gamma\left(-\frac{1}{2}\right)\right) t^{-3 / 2}+\left(b / \Gamma\left(-\frac{3}{4}\right)\right) t^{-7 / 4}, & d=3,\end{cases}
$$

with $C=\left(2^{7} / \pi\right) \exp (\gamma-2) \simeq 9.821$. To obtain (A.16) the last Tauberian theorem of table II has been used, in which terms of relative order $(\ln (t))^{-2}$ have been neglected. Below the percolation threshold, $\varepsilon<0, \Psi(z)$ is analytic in $z$ and the first two terms in eq. (A.11) do not uniquely determine the long time dependence of $\psi(t)$. If $z_{0}(\varepsilon)$ is the location of the first singularity in $\Psi(z)$ near $z=0$, then $\psi(t)$ is bounded by $\exp \left(-z_{0} t\right)$,

$$
\psi(t) \sim B(\varepsilon, t) \mathrm{e}^{-\left|z_{0}(\varepsilon)\right| t} .
$$

The fact that both $\alpha_{1}(\varepsilon)$ and $\alpha_{2}(\varepsilon)$ diverge at $\varepsilon=0$ suggests that the amplitude $B(\varepsilon, t)$ and exponent $z_{0}(\varepsilon)$ also diverge in this limit. Eqs. (A.14)-(A.18) are the results used in section 2 .

\section{Appendix B}

\section{Numerical techniques}

This appendix deals with the dimensionless variables $z, \omega, t, \Psi(z)$ and $\Psi(t)$ introduced in section 3. The asterisk is dropped for convenience. We are interested in $t$ - and $\omega$-values with $10^{-2}<t<10^{3}$ and $10^{-5}<\omega<10^{4}$.

First we calculate $\Psi(z)$ for $z=\mathrm{i} \omega$. We used a software library routine to solve the transcendental eq. (2.8) for complex $\Psi(z)$, written in the form $f(\Psi)-R(f(\Psi))=0$, with $f(\Psi)=\Psi(z)-\Psi(0)$. Another method, iteration of $f_{n+1}=R f_{n}(n=0,1,2, \ldots)$, does not converge. The velocity autocorrelation function has a singular part at $t=0$. Therefore, attention is focused on the remainder, $\Psi_{+}(z)=\Psi(z)-\Psi(\infty)$. For $t>0$ one has

$$
\psi(t)=\int_{-\mathrm{i} \infty}^{\mathrm{i} \infty} \frac{\mathrm{d} z}{2 \pi \mathrm{i}} \mathrm{e}^{z t} \Psi_{+}(z)=\frac{2}{\pi} \int_{0}^{\infty} \mathrm{d} \omega \cos (\omega t) \operatorname{Re} \Psi_{+}(\mathrm{i} \omega)
$$

By defining $\psi(t)=\psi(-t)$ for $t<0$, the inverse Laplace transform for $z=\mathrm{i} \omega$ has been expressed as a cosine transform. In the numerical evaluation of the VACF 
in the time interval specified above, we choose an appropriate $\omega_{\mathrm{m}}$ for numerical integration, beyond which we carry out the integral (semi)analytically. The simplest choice is assuming $\operatorname{Re} \Psi$ constant for $\omega>\omega_{\mathrm{m}}: \operatorname{Re} \Psi(\mathrm{i} \omega)=\operatorname{Re} \Psi\left(\mathrm{i} \omega_{\mathrm{m}}\right)$. The remaining integral has a strongly oscillating integrand for $\omega$ and $t$ sufficiently large. The small long time results $\left(t^{-2}\right.$ or $\left.\mathrm{e}^{-t}\right)$ are obtained by adding and subtracting large numbers. The simple "cutoff" choice yields results that oscillate around the expected long time behavior of $\psi(t)$. This is explained by the finite numerical accuracy in $\Psi(\mathrm{i} \omega)$, amplified by the (in general) non-continuous derivative of $\Psi$ and $\omega_{\mathrm{m}}$, induced by the constant continuation for $\omega>\omega_{\mathrm{m}}$. Only for $c$-values for which we can choose an $\omega_{\mathrm{m}}<10^{4}$ for which $\operatorname{Re} \Psi$ has approached a constant (see figs. 1a and 2a), we get good results. This numerical problem is solved by assuming for $\omega>\omega_{\mathrm{m}}$ the form

$$
\operatorname{Re} \Psi(\mathrm{i} \omega)=\alpha-\beta \omega^{-1 / 2}-\gamma \omega^{-1},
$$

where a choice for $\alpha$ determines $\beta$ and $\gamma$ through the continuity requirement for $\operatorname{Re} \Psi$ and $\mathrm{d}(\operatorname{Re} \Psi) / \mathrm{d} \omega$ at $\omega_{\mathrm{m}}$. Then the part of the integral (B.1), where $\omega>\omega_{\mathrm{m}}$, is computed as

$$
\int_{\omega_{\mathrm{m}}}^{\infty} \mathrm{d} \omega \cos (\omega t) \beta \omega^{-1 / 2}=\beta(2 \pi / t)^{1 / 2}\left\{C(\infty)-C\left(\left(2 \omega_{\mathrm{m}} t / \pi\right)^{1 / 2}\right)\right\},
$$

where $C(n)$ is the cosine Fresnel integral [23] and

$$
\int_{\omega_{\mathrm{m}}}^{\infty} \mathrm{d} \omega \cos (\omega t) \gamma \omega^{-1}=\gamma \int_{\omega_{\mathrm{m}} t}^{\infty} \mathrm{d} x \cos (x) x^{-1}=-\gamma \operatorname{Ci}\left(\omega_{\mathrm{m}} t\right),
$$

with $\mathrm{Ci}(n)$ the cosine integral [23]. Both integrals are available in software libraries.

The high frequency behavior determines the small $t$ results, so for small $t, \omega_{\mathrm{m}}$ must be chosen sufficiently large to yield results that are insensitive to $\omega_{m}$. The remaining part of the integral is performed as follows. $\Psi(z)-\Psi(0)$ is interpolated (cubic interpolation) on a mesh of points generated by a routine that is also used to produce figs. 1 and 2 . Interpolation does not work very well on $\sqrt{\omega}$-type of input. This is circumvented by applying it to the log of the function and exponentiating afterwards. Alternatively, one can divide by some elementary function that is similar to the actual one. For $\varepsilon \leqq 0$ we use $f(\omega)=$ $\alpha \omega^{2} /(\beta+\omega)^{3 / 2}$. For $\varepsilon \geqq 0$ we use $f(\omega)=\alpha \omega /(\beta+\omega)^{1 / 2} . \alpha$ and $\beta$ follow from $f^{\prime}(0)=\Psi^{\prime}(0)$ and $f\left(\omega_{\mathrm{m}}\right)=\Psi\left(\omega_{\mathrm{m}}\right)$. For $\varepsilon \geqq 0$ this method also allows subtraction techniques to compute the integral, because $\int_{0}^{\omega_{m}} d \omega \cos \omega t[\alpha \omega /(\beta+\omega)]$ 
can be expressed in terms of sine and cosine Fresnel integrals. However, careful interpolation turns out to be more effective than subtraction.

The integral

$$
\int_{0}^{\omega_{m}} \mathrm{~d} \omega \cos (\omega t) g(\omega)
$$

with $g=\operatorname{Re} \Psi$ or $g=\operatorname{Re} \Psi-\alpha \omega /(\beta+\omega)$ was evaluated using Filon's method, as given by eq. (25.4.47) of ref. [23].

\section{References}

[1] J.W. Haus and K.W. Kehr, Phys. Rep. 150 (1987) 263.

[2] S. Havlin and D. Ben-Araham, Adv. Phys. 36 (1987) 695.

[3] Y. Pomeau and P. Resibois, Phys. Rep. 19C (1975) 64.

[4] M.H. Ernst, J. Machta, J.R. Dorfman and H. van Beijeren, J. Stat. Phys. 34 (1984) 477; 35 (1984) 413.

[5] B. Alder and W.E. Alley, J. Stat. Phys. 19C (1978) 64; Physica A 121 (1983) 523.

[6] M.H. Ernst, G.A. van Velzen and J.W. Dufty, Physica A 147 (1987) 268. For an alternative explanation, see ref. [19].

[7] Th.M. Niewenhuizen, P.F.J. van Velthoven and M.H. Ernst, Phys. Rev. Lett. 57 (1986) 2477; J. Phys. A: Math. Gen. 20 (1987) 4001, 5335.

[8] M.H. Ernst, P.F.J. van Velthoven and Th.M. Niewenhuizen, J. Phys. A: Math. Gen. 20 (1987) 949; J. Stat. Phys. 45 (1986) 1001.

[9] M.H. Ernst, J. Stat. Phys. 48 (1987) 645.

G.A. van Velzen and M.H. Ernst, J. Stat. Phys. 48 (1987) 677.

[10] T. Odagaki and M. Lax, Phys. Rev. B 24 (1981) 5284.

T. Odagaki, M. Lax and A. Puri, Phys. Rev. B 28 (1983) 2755.

T. Odagaki, Phys. Rev. B 33 (1986) 544.

[11] J.W. Haus, K.W. Kehr and K. Kitahara, Phys. Rev. B 25 (1982) 4918; Z. Physik B 50 (1983) 161.

[12] I. Webman, Phys. Rev. Lett. 47 (1981) 1496.

[13] B. Derrida, R. Orbach and K-W. Yu, Phys. Rev. B 29 (1984) 6645.

[14] M. Sahimi, B.D. Hughes, L.E. Scriven and H.T. Davis, Phys. Rev. B 28 (1982) 307; J. Chem. Phys. 78 (1987) 6847.

[15] G. Ahmed and J.A. Blackman, J. Phys. C: Solid State 12 (1987) 837.

[16] D. Frenkel, Phys. Lett. A 121 (1987) 385.

[17] J.J. Brey, J. Gomez Ordonez and A. Santos, Phys. Lett. A 127 (1988) 5; and to be published.

[18] J. Straley, J. Phys. C 13 (1980) 2991.

[19] W. Goetze, E. Leutheusser and S. Yip, Phys. Rev. A 23 (1981) 2634; 24 (1981) 1008.

[20] A. Masters and T. Keyes, Phys. Rev. A 26 (1982) 2129.

T. Keyes, Phys. Rev. A 28 (1983) 2584.

E. Leutheusser, Phys. Rev. A 28 (1983) 1762.

[21] T. Vicsek, Z. Physik B: Cond. Matter 45 (1981) 153.

[22] T. Keyes, Phys. Rev. A 29 (1984) 415.

[23] M. Abramowitz and I. Stegun, Handbook of Mathematical Functions (Dover, New York, 1972). 